\title{
Enhancement of Penetration of Millimeter Waves by Field Shaping Applied to Breast Cancer Detection
}

\author{
Ioannis Iliopoulos, Simona di Meo, Student Member, IEEE, Marco Pasian, Senior Member, IEEE, \\ Maxim Zhadobov, Senior Member, IEEE, Philippe Pouliguen, Patrick Potier, Luca Perregrini, Fellow, IEEE, \\ Ronan Sauleau, Fellow, IEEE, and Mauro Ettorre, Senior Member, IEEE,
}

\begin{abstract}
Objective: The potentialities of improving the penetration of millimeter waves for breast cancer imaging are here explored. Methods: A field shaping technique based on a convex optimization method is proposed, capable of increasing the field level inside a breast-emulating stratification. Results: The theoretical results are numerically validated via the design and simulation of two circularly polarized antennas. The experimental validation of the designed antennas, using tissue-mimicking phantoms, is provided, being in good agreement with the theoretical predictions. Conclusion: The possibility of focusing, within a lossy medium, the electromagnetic power at millimeter-wave frequencies is demonstrated. Significance: Field shaping can be a key for using millimeter waves for breast cancer detection.
\end{abstract}

Index Terms-Breast Cancer, Microwave Imaging, Millimeter Waves, Radial Line Slot Arrays, Near-Field Optimization

\section{INTRODUCTION}

$\mathbf{S}$ TUDIES [1] show that in the course of her lifetime 1 of 8 women faces the risk of developing breast cancer. Additionally, breast cancer is the leading cause of cancerrelated death among females worldwide [2], and is the most common cancer in females in Europe, North America and Oceania. On the other hand, it has been shown that systematic screening [3], [4], that promotes the early diagnosis, can save lives and increase the treatment options.

Towards the direction of early diagnosis, frequent screenings are essential. Traditionally, breast imaging is performed via ultrasound, magnetic resonance imaging (MRI) or X-ray mammography, but only the latter is used in some countries

I. Iliopoulos was with the Univ Rennes, CNRS, IETR - UMR 6164, F35000 , Rennes, France. He is now with the Department of Radar Technology, Netherlands Organization for Applied Scientific Research (TNO), 2597 AK, The Hague, The Netherlands. (e-mail: yannis.iliopoulos@tno.nl).

M. Zhadobov, R. Sauleau and M. Ettorre are with the Univ Rennes, CNRS, IETR - UMR 6164, F-35000, Rennes, France (e-mail: mauro.ettorre@univrennes1.fr).

M. Pasian, S. di Meo and L. Perregrini are with the Department of Electrical, Computer and Biomedical Engineering, University of Pavia, 27100 Pavia, Italy (e-mail: marco.pasian@unipv.it).

P. Pouliguen is with the Strategy Directorate, Direction Genérale de l'Armement (DGA), Paris 75509, France (e-mail: philippe.pouliguen@intradef.gouv.fr).

P. Potier is with the Information Superiority, Direction Genérale de l'Armement (DGA), Bruz 35170, France (e-mail: patrick.potier@intradef.gouv.fr).

This work was supported in part by the Direction Générale de l'Armement and the Centre National de la Recherche Scientifique and in part by the European Union through the European Regional Development Fund, the French Region of Brittany, Ministry of Higher Education and Research, Rennes Métropole and Conseil Départemental 35 through the CPER Project STIC \& Ondes, as well as by the Italian Association for Cancer Research and the European Institute of Oncology. for screening purposes. This is because ultrasound imaging depends heavily on the ability of the physician that perform it, and it is not suited for breasts with a high fat content, while the most important drawback for MRI is its huge cost. On the other hand, X-ray mammography involves ionizing radiation and thus includes risks when it comes to frequent periodical screenings, not to mention the possible inacurracies of the technique (undetected cancers, false positives, etc.) [2].

It is apparent that a method that is safe, reliable, physicianindependent and relatively low-cost would be highly appreciated by the medical community, especially for mass periodical screenings. Consequently, in the past years several research groups have been working on the possible use of microwave systems, with few of them reaching clinical trials [5]-[10]. Nevertheless, as long as a sub-centimeter resolution is not reached, the system cannot be used to detect early-stage cancers [11], [12], and this is one of the chief reason for an interest toward other frequencies [13].

This is because current electromagnetic breast cancer imaging systems operate in the microwave frequency range. Despite the fact that some groups have reached a maximum frequency in the order of $15 \mathrm{GHz}$ [8], the achievable resolution at the central frequency $\left(\lambda_{\mathrm{fat}}^{@ 7.5 \mathrm{GHz}} / 2 \simeq 9 \mathrm{~mm}\right)$ is often not enough to detect small malignant tumors. On the other hand, following the same logic, the resolution reaches the value of $2.6 \mathrm{~mm}$ at $30 \mathrm{GHz}$ [14], allowing at least a 3-fold improvement of the imaging resolution.

In the current study, the envisioned system operates at the lower limit of millimeter waves. The central frequency is set to $30 \mathrm{GHz}$ and preliminary studies [15], [16] show very promising results. In order to increase also the range resolution a wide bandwidth is envisaged, from $20 \mathrm{GHz}$ to $40 \mathrm{GHz}$. The latter is to be defined with respect to the possible limitations of the antenna and system design.

Nevertheless, the increase of the frequency is accompanied by a significant increase in losses. This shortens substantially the effective distance at which electromagnetic waves can penetrate inside the tissue and extract useful information. In this direction a method is here proposed, capable of achieving higher field amplitudes inside the breast. This is accomplished by a focalization of the field by means of a convex optimization technique. Additionally, we set a circular polarization requirement for the radiators, which in MRI and optical imaging is shown to reduce artifacts [17] and increase the depth resolution and contrast [18].

The paper is organized as follows. In Section III the ap- 
plied optimization technique is explained and the theoretical results are presented. In Section III the practical validation is detailed, in terms of two Circularly Polarized Radial Line Slot Array (CP-RLSA) antenna prototypes. Section IV includes the measurements of the prototypes. Finally, Section $[\mathrm{V}$ draws the conclusions.

\section{InCREASING THE PENETRATION DEPTH}

\section{A. Field propagation inside the breast model}

A planar stratified breast model corresponding to the American College of Radiology (ACR) Class 1 (mostly fatty) and 2 (scattered fibroglandular) [19] was defined. The simplified model (visible in Fig. 1, dielectric properties reported in Table I), is realized on the grounds of standard assumptions for this type of situations, and it consists of $2 \mathrm{~mm}$ of skin, $40 \mathrm{~mm}$ of fat and an infinite layer of fibroglandular (FG) tissue. Taking into account that the free-space wavelength at $30 \mathrm{GHz}$ is $10 \mathrm{~mm}$, which is less than the radius of curvature of breasts, the choice of a planar model carries reasonable approximations for the present state of the research. In any case, future developments taking into account also the curvature of the breast, even if out of the scope of the present paper, are anticipated.

The breast model is illuminated by a circular aperture of radius $R=30 \mathrm{~mm}$. The size of the aperture was chosen in accordance with [15], where it is shown that a linear array of length equal to $7 \lambda(70 \mathrm{~mm})$ is the maximum beneficial size of a source from a signal-to-noise ratio (SNR) point of view. More elements (larger arrays) would contribute negatively to the total SNR of the system. As a result, we chose here a source diameter of $6 \lambda$.

We assume that the aperture supports a tangential-to-theaperture field with $\phi$-symmetry and right-hand circular polarization of the form $\boldsymbol{E}^{\mathrm{AP}}=E^{\mathrm{AP}}(\rho)(\hat{\boldsymbol{x}}-j \hat{\boldsymbol{y}})$, which generates an electric field $\boldsymbol{E}=E_{x} \hat{\boldsymbol{x}}+E_{y} \hat{\boldsymbol{y}}+E_{z} \hat{\boldsymbol{z}}$ inside the breast model. The field is evaluated inside the stratification via a spectral Green's function approach based on [23], by assuming equivalent magnetic currents at the aperture plane $\left(\boldsymbol{M}=-\hat{z} \times \boldsymbol{E}^{\mathrm{AP}}\right)$.

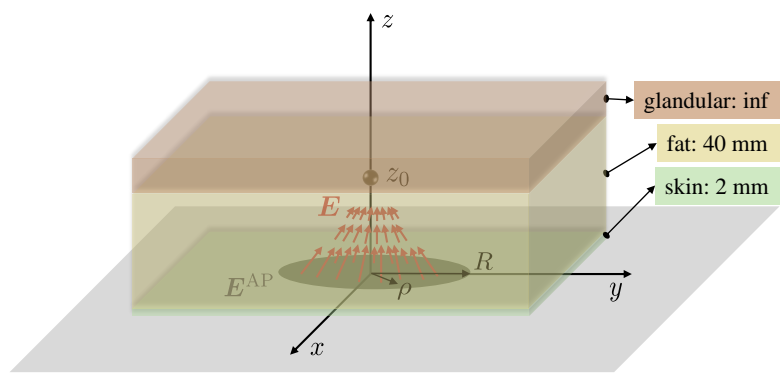

Fig. 1. Schematic of the planar breast model.

TABLE I

TISSUE PROPERTIES AT $30 \mathrm{GHZ}$ CONSIDERED FOR THE BREAST MODEL.

\begin{tabular}{l|c|c|c} 
Tissue & $\varepsilon_{r}$ & $\tan \delta$ & Reference \\
\hline \hline Skin (wet) & 17.7 & 0.93 & $\overline{20}$ \\
\hline Fat & 3.4 & 0.16 & $\overline{21}$ \\
\hline Fibroglandular & 16 & 0.94 & $\overline{22}$
\end{tabular}

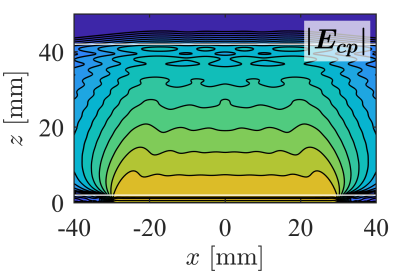

(a)

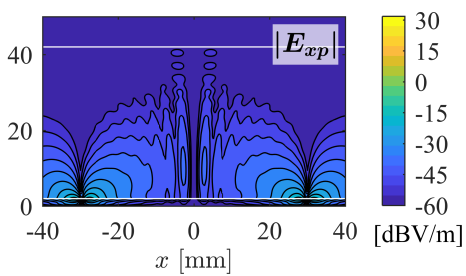

(b)
Fig. 2. (a) Co- and (b) cross-polarized $E$-field on the $x z$-plane of the breast model for a uniformly, circularly polarized source radiating $10 \mathrm{dBm}$ of power. The white lines correspond to the tissue boundaries.

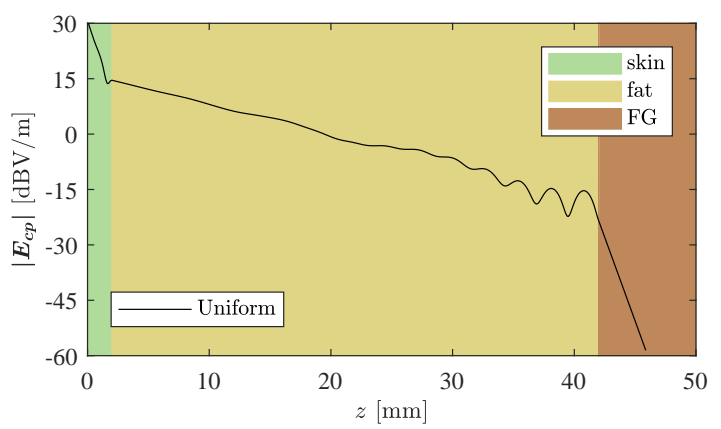

Fig. 3. Illustration of $\boldsymbol{E}_{c p}$ field inside the breast model along the $z$-axis, when the aperture is uniformly illuminated.

The near field inside the stratification has been evaluated in the case of a uniform and right-hand circularly polarized (RHCP) aperture distribution $(R=30 \mathrm{~mm})$. The field generated inside the breast model is illustrated in Fig. 2 for the two main vertical planes ( $x z$ and $y z$ ). We notice the fast attenuation of the field inside the stratification. The latter is very well illustrated also in Fig. 3. Specifically at a distance of $z=40$ $\mathrm{mm}$, the relative field level is approximately $-45 \mathrm{~dB}$ below the maximum on the skin surface.

\section{B. Convex optimization scheme}

In order to mitigate the enormous losses inside the tissue at the frequencies of interest, we propose here a focusing technique based on convex programming [24]. The idea consists in maximizing the field inside the breast model at a given depth $z_{0}$ (Fig. 1). This allows the concentration of the field at the region of interest and helps reduce its dissipation in other locations. As anticipated, the frequency of operation is $f=30 \mathrm{GHz}$, which corresponds to the central frequency of the targeted band.

By solving the field maximization problem in hand [24] for four different $z_{0}$ 's, namely $10,20,30$ and $40 \mathrm{~mm}$, we obtain four different aperture distributions, illustrated in Fig. 4 . We notice that 1) the amplitude over the aperture becomes more uniform and 2) the phase raises less rapidly as $z_{0}$ increases.

The $x z$-plane of the near fields generated from these aperture distributions are illustrated in Fig. 5 (the $y z$-plane is identical and avoided for brevity). We notice the generation of focal spots at approximately the $z$ distances for which the optimization was applied. This is not exactly the case when $z_{0}=40 \mathrm{~mm}$. This is credited to the fact that the field is heavily 

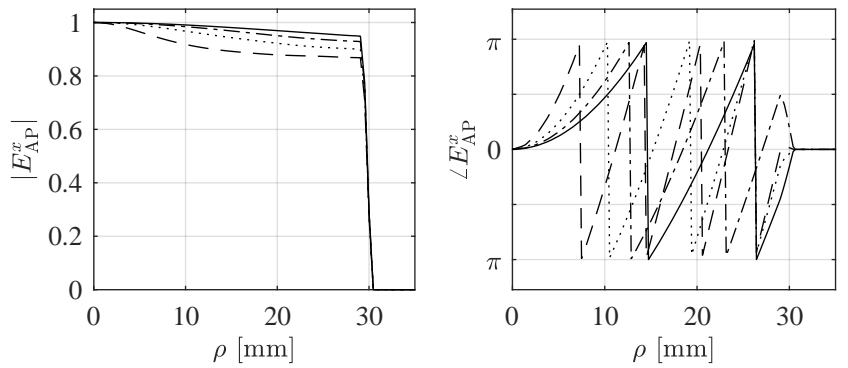

Fig. 4. Optimal aperture distributions ( $\phi$-symmetric) that maximize the field at different target distances $z_{0}$. The amplitudes are normalized to their respective maximums. Continuous line: $z_{0}=40 \mathrm{~mm}$, dash-dot: $z_{0}=30 \mathrm{~mm}$, dotted: $z_{0}=20 \mathrm{~mm}$, dashed: $z_{0}=10 \mathrm{~mm}$.

TABLE II

PERFORMANCE OF THE FOUR DISTRIBUTIONS FOR AN INPUT POWER OF $10 \mathrm{dBm}$. "FIELD ENHANCEMENT FACTOR $\Delta$ " CORRESPONDS TO THE INCREASE OF THE FIELD LEVEL AT $\left(0,0, z_{0}\right)$ WHEN COMPARED TO A FIELD RADIATED BY A UNIFORM APERTURE DISTRIBUTION OF THE SAME SIZE. $\lambda_{\text {fat }}=5.4 \mathrm{MM}(30 \mathrm{GHz})$

\begin{tabular}{l|c|c|c|c}
\multicolumn{1}{c|}{$\boldsymbol{z}_{\mathbf{0}}[\mathbf{m m}]$} & $\mathbf{1 0}$ & $\mathbf{2 0}$ & $\mathbf{3 0}$ & $\mathbf{4 0}$ \\
\hline \hline Field enhancement factor $\Delta[\mathrm{dB}]$ & 16.2 & 18.2 & 15.6 & 19.9 \\
\hline Field level $[\mathrm{dBV} / \mathrm{m}]$ & 24 & 17 & 8.5 & 0.6 \\
\hline 3dB beamwidth $[\mathrm{mm}]$ & 2.9 & 3.4 & 4.1 & 4.8 \\
\hline 3dB beamwidth $/ \lambda_{\text {fat }}$ & 0.54 & 0.64 & 0.76 & 0.88
\end{tabular}

attenuated at that point and the typical focusing behavior is not present on the vertical direction.

A comparison between these fields and the field generated by a uniform distribution (by an aperture of the same size, radiating the same power) along $z$-axis is provided in Fig. ??. It is apparent that each optimized distribution radiates a field with several dB's higher amplitude (at the points where the optimization was applied) compared to a uniformly illuminated aperture of the same radius.

In Table II the main parameters of the four distributions are recapitulated. We introduce here the field enhancement factor denoted as $\Delta$ that corresponds to the increase of the field level at $\left(0,0, z_{0}\right)$ when compared to a field radiated by a uniform aperture distribution of the same size. In the case of $z_{0}=40$ $\mathrm{mm}$, an impressive almost $20 \mathrm{~dB}$ of improvement compared to a uniformly illuminated aperture of the same size is achieved. Additionally, we may notice (Fig. ??) that this distribution offers a more uniform near field along $z$-axis. As an example of realistic scenario, the case $z_{0}=40 \mathrm{~mm}$ for the position of the tumor inside the breast is studied in the discussion to follow.

\section{Practical Implementation}

In order to experimentally validate the theoretical predictions, two circularly polarized radial line slot array (CP-RLSA) antennas have been designed using the substrate Rogers TMM10, with a permittivity of 9.8 (design value), loss tangent 0.0022 and thickness $0.635 \mathrm{~mm}$. The antennas are simply fed in terms of a coaxial probe located at their center. The first antenna (optimized), reproduces the distribution calculated by the optimization scheme for $z_{0}=40 \mathrm{~mm}$ (it will be denoted as "optimal"). On the second antenna, a uniform aperture distribution is mapped (constant phase and amplitude, it will be denoted as "uniform"). The goal is to compare their performances in the presence of tissue-emulating phantoms.

\section{A. CP-RLSA design}

The design of the antennas follows the principles detailed in [25] and an in-depth presentation will be avoided for brevity. CP-RLSA antennas consist of a spirally structured array of (typically hundredes) slots, etched on one side of a double grounded dielectric slab (parallel plate waveguide (PPW)). The slots are organized in pairs, with the counterparts being perpendicular to each another allowing the generation of the circular polarization. RLSA's are easily fed by a coaxial probe at the center of the structure which excites a Transverse Magnetic $\left(\mathrm{TM}_{0,0}^{z}\right)$ cylindrical mode inside the PPW (Fig. 1).

The design process is based on an iterative projection algorithm, where the positions and lengths of the slots are slightly altered at each iteration until convergence. The update rules are based on a complex fitting factor [25], taking also into account the radiated power with respect to the accepted power by the antennas. The radiated power should be maximzed to reduce edge effects [25]. An in-house tool based on a method of moments (MoM) [26] was adopted to fast the optimization process. The latter simulates the structure efficiently in the presence of an infinite skin layer on top of the upper slab conductor (medium where the slots radiate).

Two CP-RLSA antennas have been designed, one with the optimal and one with a uniform distribution mapped to it. The two antennas are shown in Fig. ??.

\section{B. Simulation Results}

The antennas have been simulated in the presence of the breast stratification (Fig. 1) for an input power of $10 \mathrm{dBm}$. The co- and cross-polarized field on the $x z$ vertical plane is reported in Fig. 6, for the central frequency $f=30 \mathrm{GHz}$. We can clearly notice the focusing trend of the radiated field by the optimal antenna. The field on the $y z$ plane follows similar trends and is not shown for brevity.

The field at the plane $z=z_{0}=40 \mathrm{~mm}$ is illustrated in Fig. 7 for both antennas. We notice the outstanding difference of the illumination between the two antennas. We observe a tenfold shrink of the beamwidth of the optimal antenna compared to the uniform one ( $4.6 \mathrm{~mm}$ versus $46 \mathrm{~mm}$ ). Additionally, there is a significant difference (see color scales) of the amplitude level achieved by each antenna. In fact, we observe an almost hundredfold increase of the amplitude $(19.4 \mathrm{~dB})$ as illustrated in Fig. 8. where a comparison of the co-polar component along $z$-axis is presented. This is in excellent agreement with the theoretical results reported in section II-B where continuous aperture distributions where studied and predicted a $19.9 \mathrm{~dB}$ improvement (see table III).

However, in order to obtain a better metric of the amplitude levels, we average the field level inside the $3 \mathrm{~dB}$ beamwidth for each antenna. The averaged field levels are $-11.9 \mathrm{dBV} / \mathrm{m}$ and $-27 \mathrm{dBV} / \mathrm{m}$ for the optimal and uniform antenna, respectively. This corresponds to an $18 \mathrm{~dB}$ of enhancement between the two, showing the significant level increase achieved by the optimal antenna. These values are summarized in table III. 


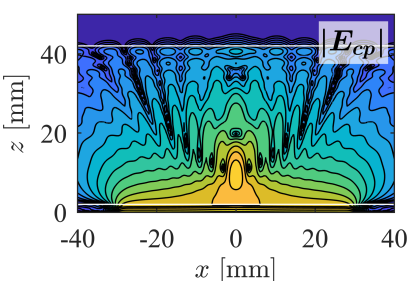

(a)

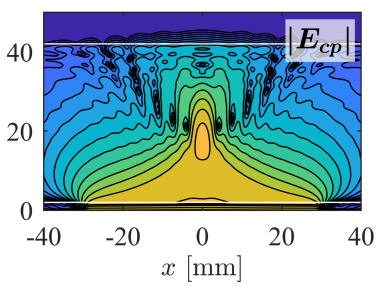

(b)

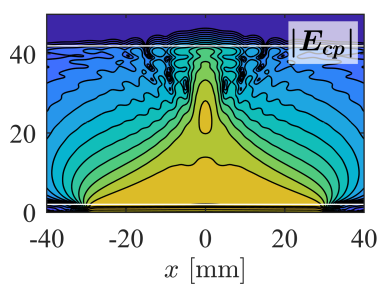

(c)

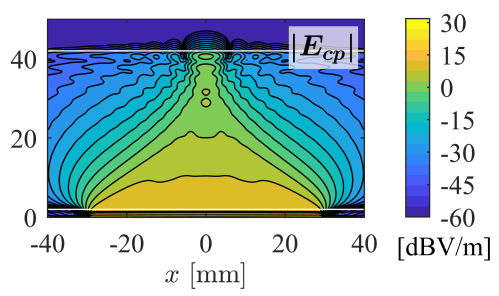

(d)

Fig. 5. Fields on the $x z$ vertical plane inside the breast model when the optimization problem is solved for (a) $z_{0}=10 \mathrm{~mm}$, (b) $z_{0}=20 \mathrm{~mm}$, (c) $z_{0}=30$ $\mathrm{mm}$ and (d) $z_{0}=40 \mathrm{~mm}$. The aperture radius is $R=30 \mathrm{~mm}$ and the radiated power is $10 \mathrm{dBm}$. Frequency $f=30 \mathrm{GHz}$. Skin starts at $z=0$ and ends at $z=2 \mathrm{~mm}$ (marked by a straight horizontal white line), fat starts at $z=2 \mathrm{~mm}$ and ends at $z=42 \mathrm{~mm}$ (marked by a second straight horizontal white line), then FG is modelled. Please note that the fields are azimuthal invariant due to the symmetry of the structure.

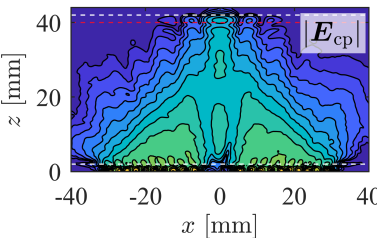

(a)

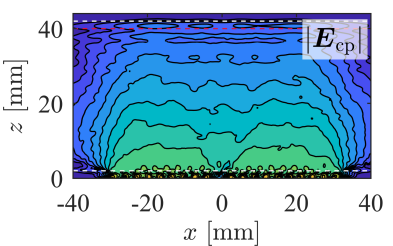

(c)

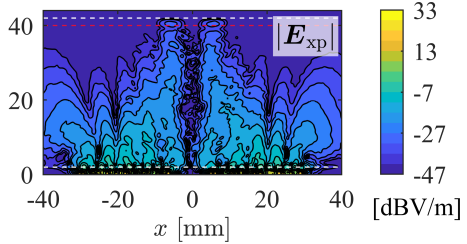

(b)

(d)

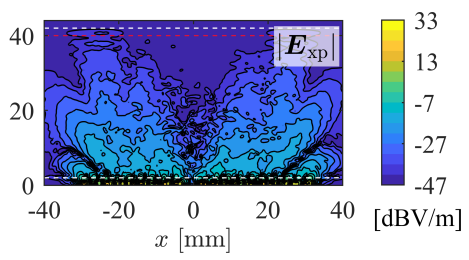

Fig. 6. $\boldsymbol{E}$-field inside the stratification at $30 \mathrm{GHz}$ along the (a) $x z$-plane and (b) $y z$-plane for the optimal antenna and (c) $x z$-plane and (d) $y z$-plane for the uniform antenna. The dashed white lines correspond to the interfaces between the tissues, as described in Fig. 5. The red dashed line corresponds to the target plane $z_{0}=40 \mathrm{~mm}$. The input power is $10 \mathrm{dBm}$.

\section{EXPERIMENTAL VALIDATION}

\section{A. Prototyping}

Both antennas were manufactured in house using laser ablation, with a metalization thickness of $17 \mu \mathrm{m}$ and a width for the slots of $70 \mu \mathrm{m}$.

Matching to the feeding connector ( $\Sigma$ RI 25-130-1000-90) is achieved by means of a disk, etched at the bottom plate of the substrate. The simulated and measured reflection coefficient is presented in Fig. 9 for both antennas. In green color, the frequency range in which the antennas are well-matched $\left(\mathrm{S}_{11}<-10 \mathrm{~dB}\right)$ are highlighted. In both cases the relative bandwidth is at the order of $50 \%$. The differences from the simulated results are accounted to the rather important sensitivity of the matching mechanism when it comes to etching precision (approx. $50 \mu \mathrm{m}$ ), as well as the sensitivity linked to the soldering applied in order to fix the connector at such frequencies. Nevertheless, the matching and the resulting operating band are very satisfying.

\section{B. Measurement set-up}

Clearly, measuring the electric field inside a medium other than air is not a common measurement practice. Consequently,

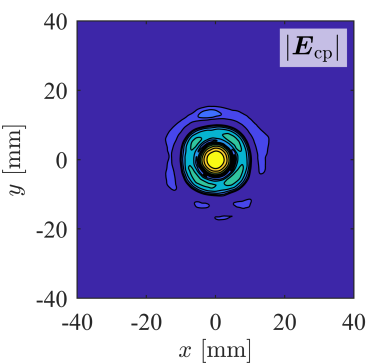

(a)

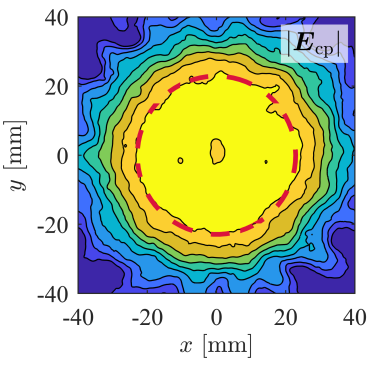

(c)

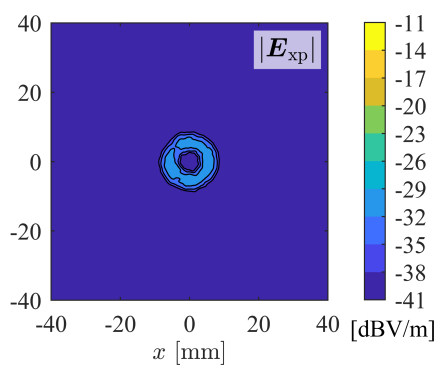

(b)

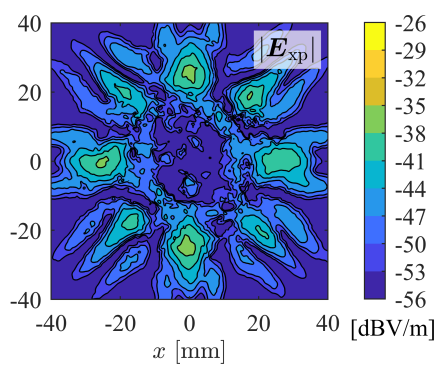

(d)
Fig. 7. Simulation results on the $z=z_{0}=40 \mathrm{~mm}$ plane. (a) and (b) co- and cross- polar component of the Optimal antenna, (c) and (d) co- and crosspolar component of the Uniform antenna. The red circle corresponds to the $3 \mathrm{~dB}$ beamwdith of the uniform antenna. The input power is $10 \mathrm{dBm}$.

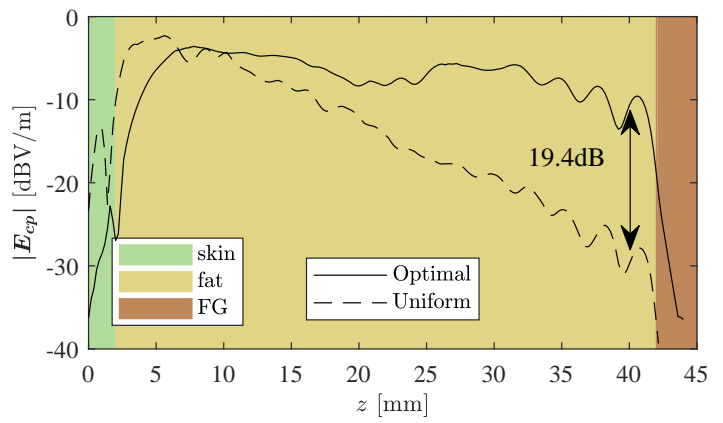

Fig. 8. Comparison along $z$-axis of the co-polar field of the optimal and uniform antenna. The results concern a $10 \mathrm{dBm}$ input power. 
1

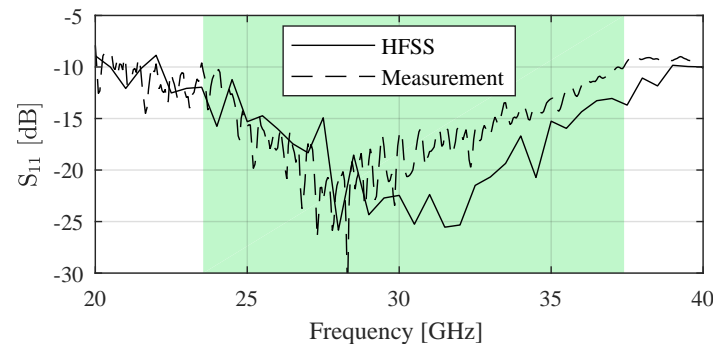

(a)

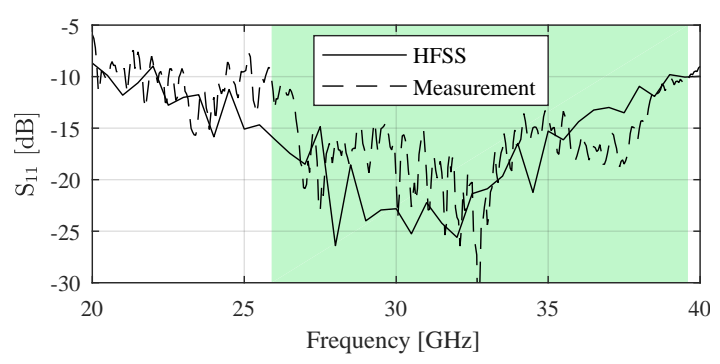

(b)

Fig. 9. Simulation results of the input reflection coefficient for the (a) optimal and (b) uniform antenna. In green color, the frequency range in which the antennas are well-matched $(\mathrm{S} 11 ;-10)$ are highlighted

we were forced to alter the model to comply with a feasible measurement set-up.

The resulting stack up is presented in Fig. 10a, where a skin and fat phantom are placed on top of the antenna. The skin and fat phantoms have thicknesses of $2 \mathrm{~mm}$ and 38 $\mathrm{mm}$ respectively, so that their assembling leads to the target distance $z_{0}=40 \mathrm{~mm}$. The measurement consists in scanning the top surface of the fat phantom using a standard WR28 open-ended probe, hence the lack of a fibroglandular layer. In more detail, the antenna with the phantom stack up is carefully positioned on a rotating base, while the probe is mounted to a 3-axis moving mast that scans the fat surface (pictures provided in Fig. 10b.

To evaluate the effect of the deviation from the original breast model, we performed further simulations in HFSS with the measurement stack up. The fields on the $z=z_{0}=40 \mathrm{~mm}$ plane are shown in Fig. 11. No significant difference is observed when it comes to the horizontal field profiles (compare with Fig. 77. The main difference is observed at the field amplitude (Fig. 12). More precisely, we notice a $17.4 \mathrm{~dB}$ (versus $19.4 \mathrm{~dB}$ ) difference at $\left(0,0, z_{0}=40 \mathrm{~mm}\right.$ ) and $15.5 \mathrm{~dB}$ (versus $18 \mathrm{~dB}$ ) when the field is averaged inside the beamwidth area. These values, which are the ones to be compared with the measurement results, are summarized in Table III] (Case: Simulation / Air on top).

\section{Measurements}

Skin and fat phantoms were realized in order to perform the measurements. The first was a mixture of gelatin in water, reported in [27]. For the fat phantom a novel procedure was developed in order to reach the target value of fat at this frequency. An emulsion of water in sunflower oil was stabilized using the emulsifying agent polysorbate 80 and then
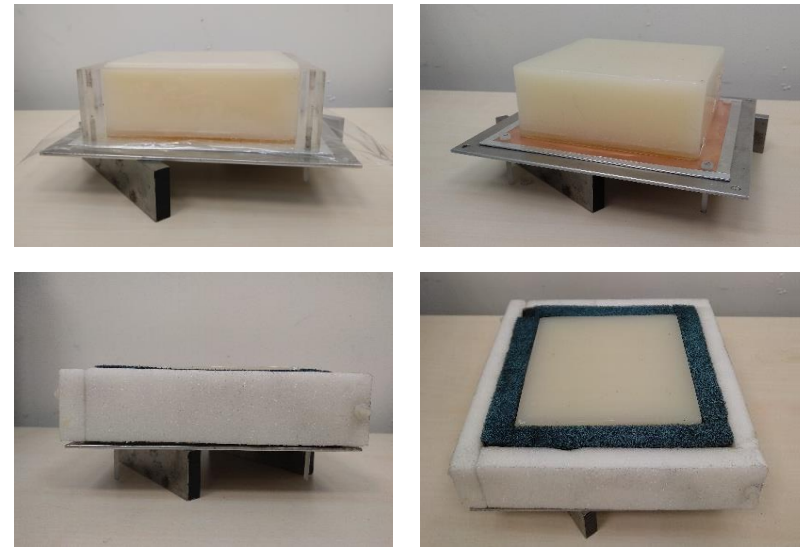

(a)
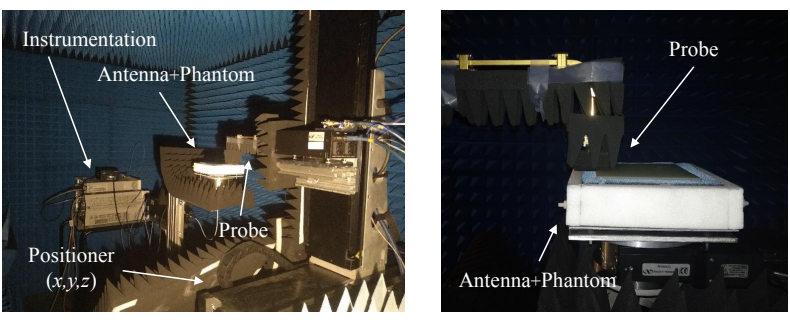

(b)

Fig. 10. (a) Preparation of the antenna and phantom stack up, (b) the measurements setup.

solidified using an oil-hardening substance (Kokubo). Further details on the preparation can be found in [28]. A $38 \mathrm{~mm}$ thick plexiglas rectangular mold was used to cast the fat phantom. The mold is visible in Fig. 10a A similar $2 \mathrm{~mm}$ thick mold was used for the skin phantom. The two phantoms were then positioned on top of the antenna as seen in Fig. 10a and covered with a border of absorbing material and foam.

The 2-D near-field scans for both antennas are visible in Fig. 13 , in terms of the co- and cross-polar components. We readily observe the focusing effect achieved by the optimal antenna (Fig. 13a), as well as the low cross-polar component (-15 dB, Fig. 13b). We might though observe a rather noisy patter especially for the uniform antenna. This is accounted to at the interface between the phantom and the absorber, as well as to the lack of perfect homogeneity of the phantoms.

Nevertheless, we observe the significantly higher level achieved in the case of the optimal antenna compared to the uniform one. Specifically, the field level at $(0,0)$ is $17.4 \mathrm{~dB}$ higher for the optimal antenna, which is in perfect agreement with the simulation results. The averaged field (along the beamwidth region) is $14.8 \mathrm{~dB}$ higher in the case of the optimal antenna, providing less than $1 \mathrm{~dB}$ deviation from the simulation results (Table IIII). The latter is due to the fact that we used the same phantom to measure both antennas, firstly for the optimal and then for the uniform, which due to evaporation presented slightly lower losses (see Fig. 14). A more detailed comparison of the field profiles is provided in Fig. 15 Taking into account the complex measurement setup, we may appreciate a very good agreement with the simulations. It is also worth mentioning that the maximum level of the measured fields in the uniform case was in the 
TABLE III

OBTAINED FIELD LEVELS AND FIELD IMPROVEMENT FACTOR $\Delta$ THROUGH SIMULATIONS AND MEASUREMENTS. IN THE CASE OF MEASUREMENTS, THE RESULTS ARE NORMALIZED TO THE MAXIMUM OF THE OPTIMAL ANTENNA. THE "AIR ON TOP” MODEL CORRESPONDS TO THE MODEL USED FOR THE MEASUREMENTS (2 MM SKIN, 38 MM FAT AND AN INFINITE LAYER OF AIR).

\begin{tabular}{|c|c|c|c|c|c|c|}
\hline Result / Model & Antenna & $\left|\boldsymbol{E}_{c p}(0,0,40 \mathrm{~mm})\right|$ & $\Delta$ & $3 \mathrm{~dB} \mathrm{BW}$ & Aver. $\left|\boldsymbol{E}_{c p}\right|$ & $\Delta$ \\
\hline \multirow{2}{*}{ Simulation / Breast (Fig 1 ) } & Optimal & $-10.5 \mathrm{dBV} / \mathrm{m}$ & \multirow[b]{2}{*}{$19.4 \mathrm{~dB}$} & $4.6 \mathrm{~mm}$ & $-11.9 \mathrm{dBV} / \mathrm{m}$ & \multirow[b]{2}{*}{$18 \mathrm{~dB}$} \\
\hline & Uniform & $-29.9 \mathrm{dBV} / \mathrm{m}$ & & $46 \mathrm{~mm}$ & $-27.9 \mathrm{dBV} / \mathrm{m}$ & \\
\hline \multirow{2}{*}{ Simulation / Air on top (Fig. 12) } & Optimal & $-9 \mathrm{dBV} / \mathrm{m}$ & \multirow{2}{*}{$17.4 \mathrm{~dB}$} & $4.7 \mathrm{~mm}$ & $-10.4 \mathrm{dBV} / \mathrm{m}$ & \multirow{2}{*}{$15.5 \mathrm{~dB}$} \\
\hline & Uniform & $-26.2 \mathrm{dBV} / \mathrm{m}$ & & $48 \mathrm{~mm}$ & $-25.9 \mathrm{dBV} / \mathrm{m}$ & \\
\hline \multirow{2}{*}{ Measurement / Air on top (Fig. 12} & Optimal & $0 \mathrm{~dB}$ & \multirow{2}{*}{$17.4 \mathrm{~dB}$} & $4.7 \mathrm{~mm}$ & $-1.6 \mathrm{~dB}$ & \multirow{2}{*}{$14.8 \mathrm{~dB}$} \\
\hline & Uniform & $-17.4 \mathrm{~dB}$ & & $48 \mathrm{~mm}$ & $-16.4 \mathrm{~dB}$ & \\
\hline
\end{tabular}

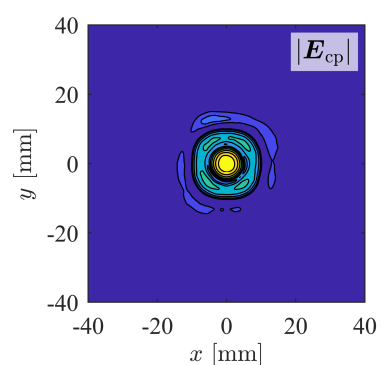

(a)

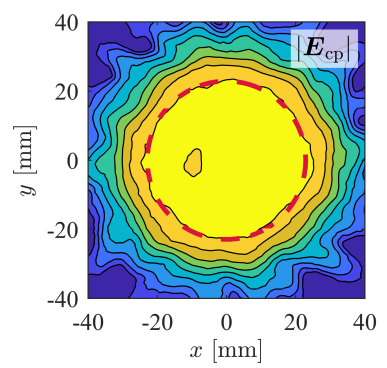

(c)

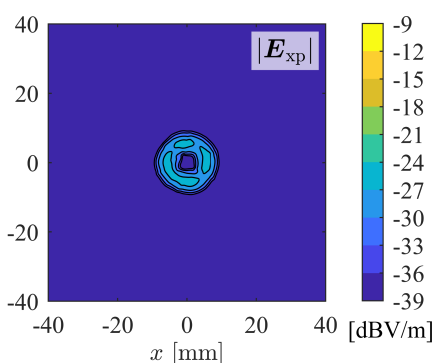

(b)

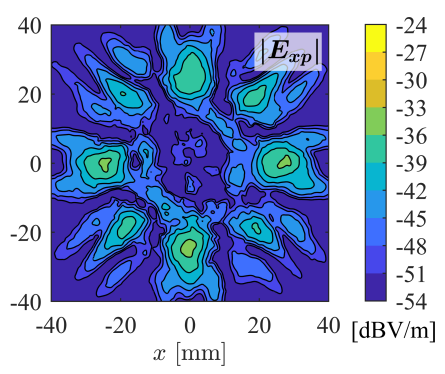

(d)
Fig. 11. Simulation results on the $z=z_{0}=40 \mathrm{~mm}$ plane for the measurement stack up. (a) and (b) co- and cross- polar component of the Optimal antenna, (c) and (d) co- and cross- polar component of the Uniform antenna. The red circle corresponds to the $3 \mathrm{~dB}$ beamwdith of the uniform antenna. The input power is $10 \mathrm{dBm}$.

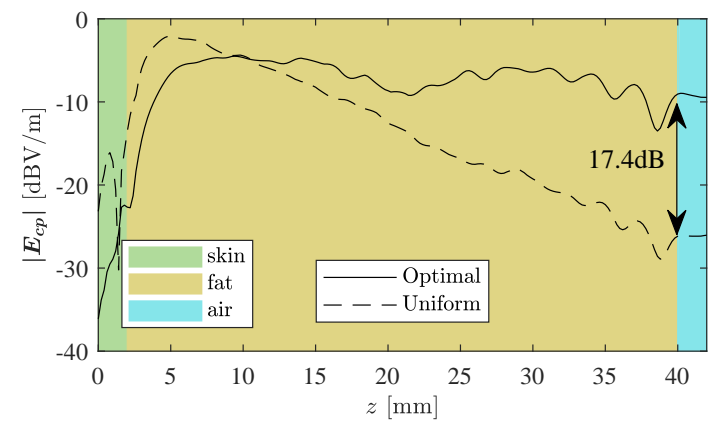

Fig. 12. Comparison along $z$-axis of the co-polar electric field of the optimal and uniform antenna inside the measurement stratification $(2 \mathrm{~mm}$ skin, 38 $\mathrm{mm}$ fat, infinite layer of air). The input power is $10 \mathrm{dBm}$.

order of $-48 \mathrm{~dB}$ (-61 dB average) with respect to the input and may justify the ripples of the radiated field observed for this case.

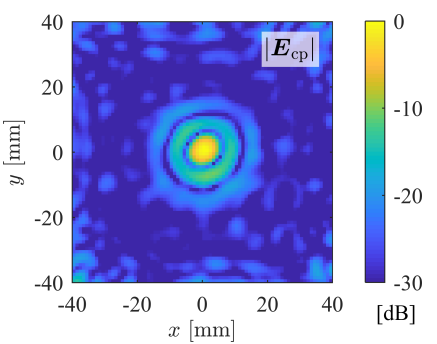

(a)

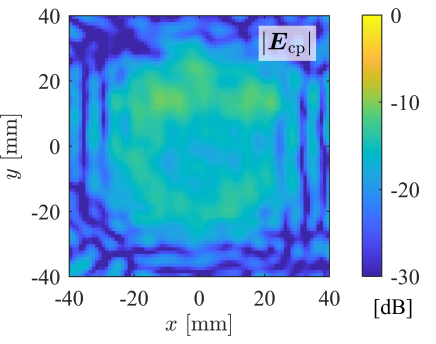

(c)

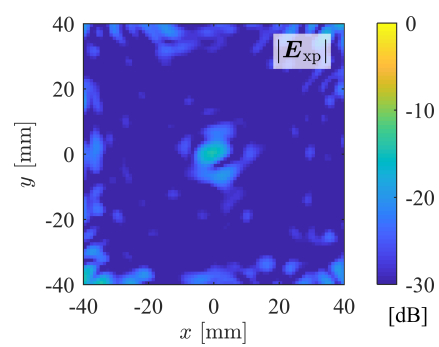

(b)

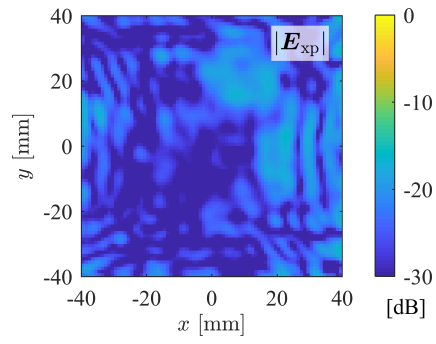

(d)
Fig. 13. Near-field measurements. (a) and (b) co- and cross- polar component of the Optimal antenna, (c) and (d) co- and cross- polar component of the Uniform antenna. The values are normalized to the maximum of the co-polar component of the optimal antenna.

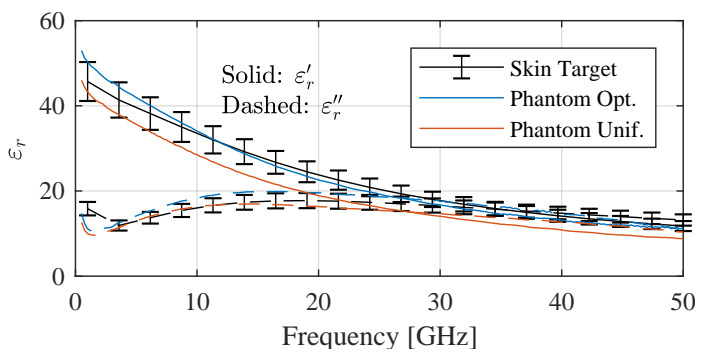

(a)

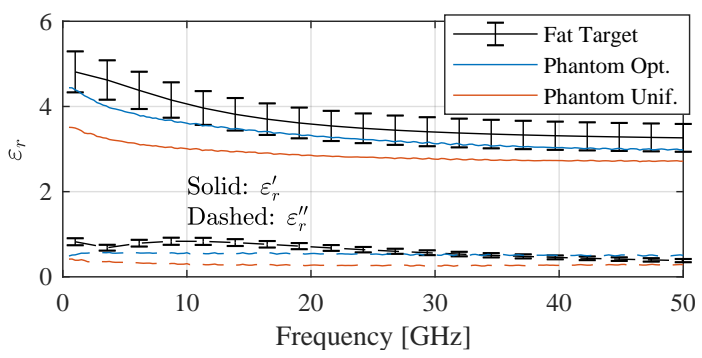

(b)

Fig. 14. Measured dielectric properties after the near-field measurements of the (a) skin and (b) fat phantom for the two antennas. 


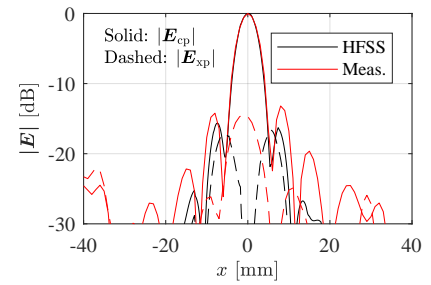

(a)

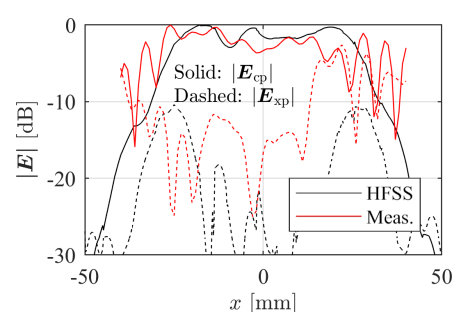

(c)

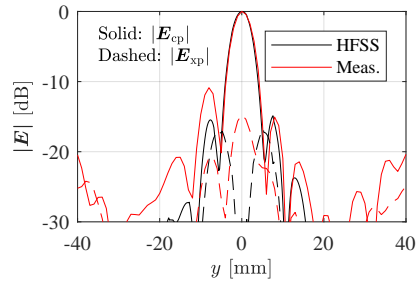

(b)

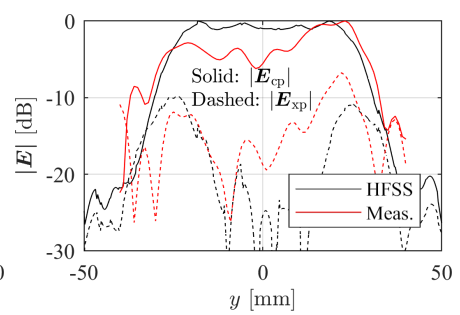

(d)
Fig. 15. Measurements versus simulations of the optimal (uniform) antenna along the (a)((c)) $x$ and (b)((d)) $y$ axis. The field is normalized to the maximum value of the co-polar component of the optimal antenna.

\section{CONClusion}

A focusing technique based on convex optimization has been used here to increase the penetration in breast cancer imaging scenarios at millimeter waves. Significant enhancements of the field level inside a breast model have been achieved by employing focused apertures, both in theory and in practice. Two CP-RLSA antennas have been designed, manufactured and measured, presenting for the first time measurements of a focused antenna in stratified media. The results are in very good agreement with the theoretical and simulation predictions, validating the overall approach. The optimized antenna presents an improvement of the field level within the breast of more than $15 \mathrm{~dB}$ with respect to uniformly radiating aperture and may provide higher sensitivity of imaging systems for cancer diagnostic at millimeter waves.

\section{ACKNOWLEDGMENT}

The authors would like to thank Rogers Corp. for the provided substrate samples. They would also like to acknowledge Benjamin Fuchs for the discussions on convex optimization, Laurent Le Coq for the near-field measurements and Rosa Orlacchio for the support during the preparation of the phantoms.

\section{REFERENCES}

[1] C. DeSantis, J. Ma, L. Bryan, and A. Jemal, "Breast cancer statistics, 2013," CA: A Cancer Journal for Clinicians, vol. 64, no. 1, pp. 52-62, Jan. 2014.

[2] R. L. Siegel, K. D. Miller, and A. Jemal, "Cancer statistics, 2016," $C A$ : A Cancer Journal for Clinicians, vol. 66, no. 1, pp. 7-30, Jan. 2016.

[3] L. E. Pace and N. L. Keating, "A systematic assessment of benefits and risks to guide breast cancer screening decisions," JAMA, vol. 311, no. 13 , pp. 1327-1335, Apr. 2014.

[4] R. A. Smith, "The value of modern mammography screening in the control of breast cancer: understanding the underpinnings of the current debates," Cancer Epidemiol Biomarkers Prev, vol. 23, no. 7, pp. 1139_ 1146, Jul. 2014.
[5] D. e. a. O'Loughlin, "Microwave breast imaging: clinical advances and remaining challenges," IEEE Transactions on Biomedical Engineering, vol. 65, no. 11, pp. 2580-2590, Nov. 2018.

[6] E. Porter, M. Coates, and M. Popovic, "An early clinical study of time-domain microwave radar for breast health monitoring," IEEE Transactions on Biomedica, vol. 63, no. 3, pp. 530-539, Mar. 2016.

[7] H. e. a. Song, "Detectability of breast tumor by a hand-held impulseradar detector: performance evaluation and pilot clinical study,' Scientific Reports, vol. 7, 2017.

[8] E. C. Fear, J. Bourqui, C. Curtis, D. Mew, B. Docktor, and C. Romano, "Microwave breast imaging with a monostatic radar-based system: a study of application to patients," IEEE Trans. Microw. Theory Techn., vol. 61, no. 5, pp. 2119-2128, May 2013.

[9] T. Henriksson, M. Klemm, D. Gibbins, J. Leendertz, T. Horseman, A. W. Preece, R. Benjamin, and I. J. Craddock, "Clinical trials of a multistatic UWB radar for breast imaging," in 2011 Loughborough Antennas Propagation Conference, Nov. 2011.

[10] P. M. Meaney, M. W. Fanning, T. Raynolds, C. J. Fox, Q. Fang, C. A. Kogel, S. P. Poplack, and K. D. Paulsen, "Initial clinical experience with microwave breast imaging in women with normal mammography," Academic Radiology, vol. 14, no. 2, pp. 207-218, Feb. 2007.

[11] N. Nikolova, "Microwave imaging for breast cancer," IEEE Microwave Mag., vol. 12, no. 7, pp. 78-94, Dec. 2011.

[12] M. Klemm, I. J. Craddock, and A. W. Preece, "Contrast-enhanced breast cancer detection using dynamic microwave imaging," in 2012 IEEE Antennas Propag. Symp. (APS 2012), Jul. 2012, pp. 1-2.

[13] F. Topfer and J. Oberhammer, "Millimeter-wave tissue diagnostics: the most promising fields for medical applications," IEEE Microwave Magazine, vol. 16, no. 4, pp. 97-113, Apr. 2015.

[14] S. Moscato, G. Matrone, M. Pasian, A. Mazzanti, M. Bozzi, L. Perregrini, F. Svelto, G. Magenes, P. Arcioni, and P. Summers, "A mmwave 2D ultra-wideband imaging radar for breast cancer detection," International Journal of Antennas and Propagation, vol. 2013, Article ID 475375,8 pages, 2013. doi:10.1155/2013/475375.

[15] S. Di Meo, P. F. Espin-Lopez, A. Martellosio, M. Pasian, G. Matrone, M. Bozzi, G. Magenes, A. Mazzanti, L. Perregrini, F. Svelto, P. E. Summers, G. Renne, L. Preda, and M. Bellomi, "On the feasibility of breast cancer imaging systems at millimeter-wave frequencies," IEEE Trans. Microw. Theory Techn., vol. 65, no. 5, pp. 1795-1806, May 2017.

[16] P. E. Summers, A. Vingiani, S. Di Pietro, A. Martellosio, P. F. EspinLopez, S. Di Meo, M. Pasian, M. Ghitti, M. Mangiacotti, R. Sacchi, M. Bozzi, A. Mazzanti, L. Perregrini, F. Svelto, L. Preda, M. Bellomi, and G. Renne, "Towards mm-wave spectroscopy for dielectric characterization of breast surgical margins," Elsevier The Breast, vol. 45, pp. 64-69, Jun. 2019.

[17] G. Glover, C. Hayes, N. Pelc, W. Edelstein, O. Mueller, H. Hart C. Hardy, M. O'Donnell, and W. Barber, "Comparison of linear and circular polarization for magnetic resonance imaging," Journal of Magnetic Resonance (1969), vol. 64, no. 2, pp. 255-270, Sep. 1985.

[18] S. Sridhar and A. Da Silva, "Enhanced contrast and depth resolution in polarization imaging using elliptically polarized light," J. Biomed. Opt., vol. 21 , no. 7 , p. 71107 , Jul. 2016.

[19] U. of Wisconsin-Madison, "Phantom repository," Sep. 2017. [Online]. Available: https://uwcem.ece.wisc.edu/phantomRepository.html

[20] P. Hasgall, F. Di Gennaro, C. Baumgartner, E. Neufeld, M. C. Gosselin, D. Payne, A. Klingenböck, and N. Kuster, "IT'IS Database for thermal and electromagnetic parameters of biological tissues," Sep. 2015.

[21] M. Lazebnik, L. McCartney, D. Popovic, C. Watkins, M. Lindstrom, J. Harter, S. Sewall, A. Magliocco, J. Booske, M. Okoniewski, and S. Hagness, "A large-scale study of the ultrawideband microwave dielectric properties of normal breast tissue obtained from reduction surgeries," Physics in Medicine and Biology, vol. 52, no. 10, pp. 26372656, 2007.

[22] A. Martellosio, M. Pasian, M. Bozzi, L. Perregrini, A. Mazzanti, F. Svelto, P. E. Summers, G. Renne, L. Preda, and M. Bellomi, "Dielectric properties characterization from 0.5 to $50 \mathrm{GHz}$ of breast cancer tissues," IEEE Trans. Microw. Theory Techn., vol. 65, no. 3, pp. 998-1011, Mar. 2017.

[23] K. Michalski and J. Mosig, "Multilayered media Green's functions in integral equation formulations," IEEE Trans. Antennas Propagation, vol. 45, no. 3, pp. 508-519, Mar. 1997.

[24] I. Iliopoulos, B. Fuchs, R. Sauleau, P. Pouliguen, P. Potier, and M. Ettorre, "Scalar near-field focusing in lossy media," in 2017 Int. Conf. on Electromagn. in Adv. Appl. (ICEAA), Sep. 2017, pp. 718-721.

[25] M. Albani, A. Mazzinghi, and A. Freni, "Automatic design of CP-RLSA antennas," IEEE Trans. Antennas Propag., vol. 60, no. 12, pp. 55385547, Dec. 2012. 
[26] M. Casaletti, G. Valerio, J. Seljan, M. Ettorre, and R. Sauleau, "A full-wave hybrid method for the analysis of multilayered SIW-based antennas," IEEE Trans. Antennas Propag., vol. 61, no. 11, pp. 55755588, Nov. 2013.

[27] R. Aminzadeh, M. Saviz, and A. A. Shishegar, "Theoretical and experimental broadband tissue-equivalent phantoms at microwave and millimetre-wave frequencies," Electron. Lett., vol. 50, no. 8, pp. 618620, Apr. 2014.

[28] S. D. Meo, L. Pasotti, I. Iliopoulos, M. Pasian, M. Ettorre, M. Zhadobov, and G. Matrone, "Tissue-mimicking materials for breast phantoms up to $50 \mathrm{GHz}$," Physics in Medicine \& Biology, vol. 64, no. 5, p. 055006 , Feb. 2019. 